The authors highlight that individualization of therapy on the basis of patients' genetic features could revolutionize medicine, and conclude that their findings suggest the potential for such personalization in patients with $\mathrm{BPH}$.

Original article Kojima Y et al. (2008) Expression of $\alpha_{1}$-adrenoceptor subtype mRNA as a predictor of the efficacy of subtype selective $\alpha_{1}$-adrenoceptor antagonists in the management of benign prostatic hyperplasia. J Urol 179: 1040-1046

\section{Clinical value of ultrasonography in diagnosis of renal tuberculosis}

The value of ultrasonography for diagnosing renal tuberculosis is controversial, with several published reports showing notably varied results. Nonetheless, B-mode ultrasonography is widely used for this purpose on account of its convenience, low cost and noninvasive nature. Rui and colleagues retrospectively analyzed ultrasound images from 258 patients with renal tuberculosis, and evaluated the clinical value of ultrasonography for diagnosing and classifying this disease.

The patients (mean age 45.3 years, range 17-73 years) were diagnosed between 1993 and 2005; 120 and 118 patients had left and right kidney tuberculosis, respectively, and the remaining 20 patients had bilateral kidney tuberculosis. Some patients, in addition to ultrasonography, underwent one or more other investigations to confirm their diagnosis, including intravenous urography, retrograde pyeloureterography, CT and cystoscopy.

Analysis of ultrasound images correctly diagnosed renal tuberculosis in $152(58.9 \%)$ of 258 patients. In comparison, 113 (46.1\%) of 245 patients who underwent intravenous urography or retrograde pyeloureterography and $87(63.0 \%)$ of 138 patients who underwent CT were correctly diagnosed. The authors defined six pathologic subtypes of renal tuberculosis on the basis of specific features of the ultrasound images, which improved clinical diagnosis and also facilitated selection of an appropriate treatment strategy.

The authors conclude that ultrasonography has definite advantages for the diagnosis of renal tuberculosis, although multimodal diagnostic work-up is still required to confirm the presence of this disease and avoid misdiagnosis. Furthermore, ultrasonography is particularly useful for monitoring disease progression in patients receiving antituberculosis chemotherapy.

Original article Rui X et al. (2008) Ultrasonographic diagnosis and typing of renal tuberculosis. Int J Urol 15: 135-139

\section{Finasteride chemoprevention might be cost-effective in high-risk populations}

The Prostate Cancer Prevention Trial showed that finasteride chemoprevention reduced the 7-year prevalence of prostate cancer compared with placebo. A previous study, however, showed that widespread use of finasteride chemoprevention would be extremely expensive, with a cost of US $\$ 578,400-1,107,000$ per life-year saved (well above the generally accepted threshold of US $\$ 50,000-100,000$ per life-year saved). Svatek and colleagues, the authors of that study, have performed another model-based analysis, this time to examine the cost-effectiveness of finasteride chemoprevention in terms of the quality-of-life improvements associated with the prevention of prostate cancer.

Their Markov decision-analysis model used data from the Prostate Cancer Prevention Trial and the Surveillance, Epidemiology, and End-Results program to compare the lifetime prostate-health-associated costs for men (starting at 50 years of age) who receive finasteride chemoprevention or placebo. The main outcome measure was the cost per quality-adjusted life-year (QALY) saved.

The cost-effectiveness ratio of finasteride chemoprevention, assuming that this treatment reduces overall prostate cancer incidence with an increased incidence of high-grade prostate cancer, was US\$122,747 per QALY saved. However, sensitivity analyses showed that, in a high-risk population (prostate cancer prevalence $\geq 30 \%$ in men aged 50 years or older), the cost-effectiveness ratio of chemoprevention was consistently below the upper threshold limit (i.e. <US\$100,000 per QALY saved).

The authors conclude that finasteride chemoprevention might be cost-effective in populations at high risk of prostate cancer, so long as quality-of-life adjustments are considered in the calculations.

Original article Svatek RS et al. (2008) Cost-effectiveness of prostate cancer chemoprevention: a quality of life-years analysis. Cancer 112: 1058-1065 